\title{
Research Article \\ Bounds for Certain New Integral Inequalities on Time Scales
}

\author{
Wei Nian Li ${ }^{1,2}$ \\ ${ }^{1}$ Department of Applied Mathematics, Shanghai Normal University, Shanghai 200234, China \\ ${ }^{2}$ Department of Mathematics, Binzhou University, Shandong 256603, China \\ Correspondence should be addressed to Wei Nian Li, wnli@263.net
}

Received 31 March 2009; Accepted 10 June 2009

Recommended by Victoria Otero-Espinar

Our aim in this paper is to investigate some new integral inequalities on time scales, which provide explicit bounds on unknown functions. Our results unify and extend some integral inequalities and their corresponding discrete analogues. The inequalities given here can be used as handy tools to study the properties of certain dynamic equations on time scales.

Copyright ( $) 2009$ Wei Nian Li. This is an open access article distributed under the Creative Commons Attribution License, which permits unrestricted use, distribution, and reproduction in any medium, provided the original work is properly cited.

\section{Introduction}

The study of dynamic equations on time scales, which goes back to its founder Hilger [1], is an area of mathematics that has recently received a lot of attention. For example, we refer the reader to literatures [2-8] and the references cited therein. At the same time, some fundamental integral inequalities used in analysis on time scales have been extended by many authors [9-14]. In this paper, we investigate some new nonlinear integral inequalities on time scales, which unify and extend some continuous inequalities and their corresponding discrete analogues. Our results can be used as handy tools to study the properties of certain dynamic equations on time scales.

Throughout this paper, a knowledge and understanding of time scales and time scale notation is assumed. For an excellent introduction to the calculus on time scales, we refer the reader to monographes $[2,3]$.

\section{Main Results}

In what follows, $\mathbb{R}$ denotes the set of real numbers, $\mathbb{R}_{+}=[0, \infty), \mathbb{Z}$ denotes the set of integers, $\mathbb{N}_{0}=\{0,1,2, \ldots\}$ denotes the set of nonnegative integers, $C(M, S)$ denotes the class of all continuous functions defined on set $M$ with range in the set $S, \mathbb{T}$ is an arbitrary time scale, 
$\mathrm{C}_{\mathrm{rd}}$ denotes the set of rd-continuous functions, $R$ denotes the set of all regressive and $\mathrm{rd}$ continuous functions, and $\mathcal{R}^{+}=\{p \in R: 1+\mu(t) p(t)>0, \forall t \in \mathbb{T}\}$. We use the usual conventions that empty sums and products are taken to be 0 and 1, respectively. Throughout this paper, we always assume that $p \geq 1,0<q \leq p, p$, and $q$ are real constants, and $t \geq t_{0}, t_{0} \in \mathbb{T}^{\kappa}$.

We firstly introduce the following lemmas, which are useful in our main results.

Lemma 2.1 ([15] (Bernoulli's inequality)). Let $0<\alpha \leq 1$ and $x>-1$. Then $(1+x)^{\alpha} \leq 1+\alpha x$.

Lemma 2.2 ([2]). Let $t_{0} \in \mathbb{T}^{\kappa}$ and $w: \mathbb{T} \times \mathbb{T}^{\kappa} \rightarrow \mathbb{R}$ be continuous at $(t, t)$, where $t \geq t_{0}, t \in \mathbb{T}^{\kappa}$ with $t>t_{0}$. Assume that $w^{\Delta}(t, \cdot)$ is rd-continuous on $\left[t_{0}, \sigma(t)\right]$. If for any $\varepsilon>0$, there exists a neighborhood $U$ of $t$, independent of $\tau \in\left[t_{0}, \sigma(t)\right]$, such that

$$
\left|w(\sigma(t), \tau)-w(s, \tau)-w^{\Delta}(t, \tau)(\sigma(t)-s)\right| \leq \varepsilon|\sigma(t)-s|, \quad \forall s \in U,
$$

where $w^{\Delta}$ denotes the derivative of $w$ with respect to the first variable, then

$$
g(t):=\int_{t_{0}}^{t} w(t, \tau) \Delta \tau
$$

implies

$$
g^{\Delta}(t)=\int_{t_{0}}^{t} w^{\Delta}(t, \tau) \Delta \tau+w(\sigma(t), t)
$$

Lemma 2.3 ([2] (Comparison Theorem)). Suppose $u, b \in C_{\mathrm{rd}}, a \in \mathcal{R}^{+}$. Then

$$
u^{\Delta}(t) \leq a(t) u(t)+b(t), \quad t \geq t_{0}, t \in \mathbb{T}^{\kappa}
$$

implies

$$
u(t) \leq u\left(t_{0}\right) e_{a}\left(t, t_{0}\right)+\int_{t_{0}}^{t} e_{a}(t, \sigma(\tau)) b(\tau) \Delta \tau, \quad t \geq t_{0}, t \in \mathbb{T}^{\kappa}
$$

Lemma 2.4 (see [13]). Let $u, f, g \in C_{\mathrm{rd}}, u(t), f(t)$, and $g(t)$ be nonnegative. If $f(t)$ is nondecreasing, then

$$
u(t) \leq f(t)+\int_{t_{0}}^{t} g(\tau) u(\tau) \Delta \tau, \quad t \in \mathbb{T}^{\kappa},
$$

implies

$$
u(t) \leq f(t) e_{g}\left(t, t_{0}\right), \quad t \in \mathbb{T}^{\mathcal{K}},
$$


Next, we establish our main results.

Theorem 2.5. Assume that $u, a, b, g, h \in C_{\mathrm{rd}}, a(t)>0, u(t), b(t), g(t)$ and $h(t)$ are nonnegative. Then

$$
u^{p}(t) \leq a(t)+b(t) \int_{t_{0}}^{t}\left[g(\tau) u^{q}(\tau)+h(\tau)\right] \Delta \tau, \quad t \in \mathbb{T}^{\kappa},
$$

implies

$$
u(t) \leq a^{1 / p}(t)+\frac{1}{p} a^{1 / p-1}(t) b(t) \int_{t_{0}}^{t} e_{B}(t, \sigma(\tau)) F(\tau) \Delta \tau, \quad t \in \mathbb{T}^{\mathcal{K}},
$$

where

$$
\begin{aligned}
& F(t)=g(t) a^{q / p}(t)+h(t), \\
& B(t)=\frac{q}{p} a^{q / p-1}(t) b(t) g(t), \quad t \in \mathbb{T}^{\kappa} .
\end{aligned}
$$

Proof. Define a function $z(t)$ by

$$
z(t)=\int_{t_{0}}^{t}\left[g(\tau) u^{q}(\tau)+h(\tau)\right] \Delta \tau .
$$

Then (2.8) can be restated as

$$
u^{p}(t) \leq a(t)+b(t) z(t)=a(t)\left(1+\frac{b(t) z(t)}{a(t)}\right)
$$

Using Lemma 2.1, from the above inequality, we easily obtain

$$
\begin{aligned}
u(t) & \leq a^{1 / p}(t)+\frac{1}{p} a^{1 / p-1}(t) b(t) z(t), \\
u^{q}(t) & \leq a^{q / p}(t)+\frac{q}{p} a^{q / p-1}(t) b(t) z(t) .
\end{aligned}
$$

It follows from (2.12) and (2.15) that

$$
\begin{aligned}
z^{\Delta}(t) & =g(t) u^{q}(t)+h(t) \\
& \leq g(t)\left(a^{q / p}(t)+\frac{q}{p} a^{q / p-1}(t) b(t) z(t)\right)+h(t) \\
& =F(t)+B(t) z(t), \quad t \in \mathbb{T}^{\kappa},
\end{aligned}
$$


where $F(t)$ and $B(t)$ are defined as in (2.10) and (2.11), respectively. Using Lemma 2.3 and noting $z\left(t_{0}\right)=0$, from (2.16) we have

$$
z(t) \leq \int_{t_{0}}^{t} e_{B}(t, \sigma(\tau)) F(\tau) \Delta \tau, \quad t \in \mathbb{T}^{\kappa}
$$

Therefore, the desired inequality (2.9) follows from (2.14) and (2.17). This completes the proof of Theorem 2.5.

Corollary 2.6. Assume that $u, g \in C_{\mathrm{rd}}, u(t)$, and $g(t)$ are nonnegative. If $\alpha>0$ is a constant, then

$$
u^{p}(t) \leq \alpha+\int_{t_{0}}^{t} g(\tau) u^{q}(\tau) \Delta \tau, \quad t \in \mathbb{T}^{\mathcal{K}}
$$

implies

$$
u(t) \leq \alpha^{1 / p}\left(1-\frac{1}{q}+\frac{1}{q} e_{\widehat{B}}\left(t, t_{0}\right)\right), \quad t \in \mathbb{T}^{\kappa},
$$

where

$$
\widehat{B}(t)=\frac{q}{p} \alpha^{q / p-1} g(t), \quad t \in \mathbb{T}^{\kappa}
$$

Proof. Letting $a(t)=\alpha, b(t)=1$, and $h(t)=0$ in Theorem 2.5, we obtain

$$
F(t)=\alpha^{q / p} g(t), \quad B(t)=\frac{q}{p} \alpha^{q / p-1} g(t):=\widehat{B}(t), \quad t \in \mathbb{T}^{\mathcal{K}}
$$

Therefore,

$$
\begin{aligned}
u(t) & \leq \alpha^{1 / p}+\frac{1}{p} \alpha^{1 / p-1} \int_{t_{0}}^{t} e_{\widehat{B}}(t, \sigma(\tau)) F(\tau) \Delta \tau \\
& =\alpha^{1 / p}+\frac{1}{p} \alpha^{1 / p-1} \int_{t_{0}}^{t} e_{\widehat{B}}(t, \sigma(\tau)) \alpha^{q / p} g(\tau) \Delta \tau
\end{aligned}
$$




$$
\begin{aligned}
& =\alpha^{1 / p}+\frac{1}{q} \alpha^{1 / p} \int_{t_{0}}^{t} e_{\widehat{B}}(t, \sigma(\tau)) \frac{q}{p} \alpha^{q / p-1} g(\tau) \Delta \tau \\
& =\alpha^{1 / p}+\frac{1}{q} \alpha^{1 / p} \int_{t_{0}}^{t} e_{\widehat{B}}(t, \sigma(\tau)) \widehat{B}(\tau) \Delta \tau \\
& =\alpha^{1 / p}+\frac{1}{q} \alpha^{1 / p}\left(e_{\widehat{B}}\left(t, t_{0}\right)-e_{\widehat{B}}(t, t)\right) \\
& =\alpha^{1 / p}+\frac{1}{q} \alpha^{1 / p} e_{\widehat{B}}\left(t, t_{0}\right)-\frac{1}{q} \alpha^{1 / p} \\
& =\alpha^{1 / p}\left(1-\frac{1}{q}+\frac{1}{q} e_{\widehat{B}}\left(t, t_{0}\right)\right), \quad t \in \mathbb{T}^{\mathcal{K}} .
\end{aligned}
$$

The proof of Corollary 2.6 is complete.

Remark 2.7. The result of Theorem 2.5 holds for an arbitrary time scale. Therefore, using Theorem 2.5, we can obtain many results for some peculiar time scales. For example, letting $\mathbb{T}=\mathbb{R}$ and $\mathbb{T}=\mathbb{Z}$, respectively, we have the following two results.

Corollary 2.8. Let $\mathbb{T}=\mathbb{R}$ and assume that $u(t), a(t), b(t), g(t), h(t) \in C\left(\mathbb{R}_{+}, \mathbb{R}_{+}\right)$, and $a(t)>0$. Then the inequality

$$
u^{p}(t) \leq a(t)+b(t) \int_{0}^{t}\left[g(s) u^{q}(s)+h(s)\right] \mathrm{d} s, \quad t \in \mathbb{R}_{+}
$$

implies

$$
u(t) \leq a^{1 / p}(t)+\frac{1}{p} a^{1 / p-1}(t) b(t) \int_{0}^{t} F(\theta) \exp \left(\int_{\theta}^{t} B(s) \mathrm{d} s\right) \mathrm{d} \theta, \quad t \in \mathbb{R}_{+},
$$

where $F(t)$ and $B(t)$ are defined as in Theorem 2.5.

Corollary 2.9. Let $\mathbb{T}=\mathbb{Z}$ and assume that $a(t)>0, u(t), b(t), g(t)$, and $h(t)$ are nonnegative functions defined for $t \in \mathbb{N}_{0}$. Then the inequality

$$
u^{p}(t) \leq a(t)+b(t) \sum_{s=0}^{t-1}\left[g(s) u^{q}(s)+h(s)\right], \quad t \in \mathbb{N}_{0}
$$

implies

$$
u(t) \leq a^{1 / p}(t)+\frac{1}{p} a^{1 / p-1}(t) b(t) \sum_{\theta=0}^{t-1} F(\theta) \prod_{s=\theta+1}^{t-1}[1+B(s)], \quad t \in \mathbb{N}_{0}
$$

where $F(t)$ and $B(t)$ are defined as in Theorem 2.5. 
Investigating the proof procedure of Theorem 2.5 carefully, we easily obtain the following more general result.

Theorem 2.10. Assume that $u, a, b, g_{i}, h_{i} \in C_{\mathrm{rd}}, a(t)>0, u(t), b(t), g_{i}(t)$, and $h_{i}(t)$ are nonnegative, $i=1,2, \ldots, n$. If there exists a series of positive real numbers $q_{1}, q_{2}, \ldots, q_{n}$ such that $p \geq q_{i}>0, i=1,2, \ldots, n$, then

$$
u^{p}(t) \leq a(t)+b(t) \sum_{i=1}^{n} \int_{t_{0}}^{t}\left[g_{i}(\tau) u^{q_{i}}(\tau)+h_{i}(\tau)\right] \Delta \tau, \quad t \in \mathbb{T}^{\kappa},
$$

implies

$$
u(t) \leq a^{1 / p}(t)+\frac{1}{p} a^{1 / p-1}(t) b(t) \int_{t_{0}}^{t} e_{B^{*}}(t, \sigma(\tau)) F^{*}(\tau) \Delta \tau, \quad t \in \mathbb{T}^{\kappa},
$$

where

$$
F^{*}(t)=\sum_{i=1}^{n}\left(g_{i}(t) a^{q_{i} / p}(t)+h_{i}(t)\right), \quad B^{*}(t)=b(t) \sum_{i=1}^{n} \frac{q_{i}}{p} a^{q_{i} / p-1}(t) g_{i}(t)
$$

Theorem 2.11. Assume that $u, a, b, f, g, m \in C_{\mathrm{rd}}, a(t)>0, u(t), b(t), f(t), g(t)$ and $m(t)$ are nonnegative. If $w(t, s)$ is defined as in Lemma 2.2 such that $w(t, s) \geq 0$ and $w^{\Delta}(t, s) \geq 0$ for $t, s \in \mathbb{T}$ with $s \leq t$, then

$$
u^{p}(t) \leq a(t)+b(t) \int_{t_{0}}^{t} w(t, \tau)\left[f(\tau) u^{p}(\tau)+g(\tau) u^{q}(\tau)+m(\tau)\right] \Delta \tau, \quad t \in \mathbb{T}^{\kappa},
$$

implies

$$
u(t) \leq a^{1 / p}(t)+\frac{1}{p} a^{1 / p-1}(t) b(t) \int_{t_{0}}^{t} e_{A}(t, \sigma(\tau)) G(\tau) \Delta \tau, \quad t \in \mathbb{T}^{\kappa},
$$

where

$$
\begin{aligned}
A(t)= & w(\sigma(t), t) b(t)\left(f(t)+\frac{q}{p} a^{q / p-1}(t) g(t)\right) \\
& +\int_{t_{0}}^{t} w^{\Delta}(t, \tau) b(\tau)\left(f(\tau)+\frac{q}{p} a^{q / p-1}(\tau) g(\tau)\right) \Delta \tau, \\
G(t)= & w(\sigma(t), t)\left[a(t) f(t)+g(t) a^{q / p}(t)+m(t)\right] \\
& +\int_{t_{0}}^{t} w^{\Delta}(t, \tau)\left[a(\tau) f(\tau)+g(\tau) a^{q / p}(\tau)+m(\tau)\right] \Delta \tau .
\end{aligned}
$$


Proof. Define a function $z(t)$ by

$$
z(t)=\int_{t_{0}}^{t} w(t, \tau)\left[f(\tau) u^{p}(\tau)+g(\tau) u^{q}(\tau)+m(\tau)\right] \Delta \tau, \quad t \in \mathbb{T}^{\kappa}
$$

Then $z\left(t_{0}\right)=0$. As in the proof of Theorem 2.5, we easily obtain (2.14) and (2.15). Using Lemma 2.2 and combining (2.34) and (2.15), we have

$$
\begin{aligned}
z^{\Delta}(t)= & w(\sigma(t), t)\left[f(t) u^{p}(t)+g(t) u^{q}(t)+m(t)\right] \\
& +\int_{t_{0}}^{t} w^{\Delta}(t, \tau)\left[f(\tau) u^{p}(\tau)+g(\tau) u^{q}(\tau)+m(\tau)\right] \Delta \tau \\
\leq & w(\sigma(t), t)\left[a(t) f(t)+g(t) a^{q / p}(t)+m(t)+b(t)\left(f(t)+\frac{q}{p} a^{q / p-1}(t) g(t)\right) z(t)\right] \\
& +\int_{t_{0}}^{t} w^{\Delta}(t, \tau)\left[a(\tau) f(\tau)+g(\tau) a^{q / p}(\tau)+m(\tau)\right. \\
& \left.\quad+b(\tau)\left(f(\tau)+\frac{q}{p} a^{q / p-1}(t) g(\tau)\right) z(\tau)\right] \Delta \tau \\
\leq & {\left[w(\sigma(t), t) b(t)\left(f(t)+\frac{q}{p} a^{q / p-1}(t) g(t)\right)\right.} \\
& \left.+\int_{t_{0}}^{t} w^{\Delta}(t, \tau) b(\tau)\left(f(\tau)+\frac{q}{p} a^{q / p-1}(t) g(\tau)\right) \Delta \tau\right] z(t) \\
& +w(\sigma(t), t)\left[a(t) f(t)+g(t) a^{q / p}(t)+m(t)\right] \\
& +\int_{t_{0}}^{t} w^{\Delta}(t, \tau)\left[a(\tau) f(\tau)+g(\tau) a^{q / p}(\tau)+m(\tau)\right] \Delta \tau \\
= & A(t) z(t)+G(t), \quad t \in \mathbb{T}^{\kappa},
\end{aligned}
$$

where $A(t)$ and $G(t)$ are defined as in (2.32) and (2.33), respectively. Therefore, in the above inequality, using Lemma 2.3 and noting $z\left(t_{0}\right)=0$, we get

$$
z(t) \leq \int_{t_{0}}^{t} e_{A}(t, \sigma(\tau)) G(\tau) \Delta \tau, \quad t \in \mathbb{T}^{\kappa}
$$

It is easy to see that the desired inequality (2.31) follows from (2.14) and (2.36). This completes the proof of Theorem 2.11. 
Corollary 2.12. Let $\mathbb{T}=\mathbb{R}$ and assume that $u(t), a(t), b(t), f(t), g(t), m(t) \in C\left(\mathbb{R}_{+}, \mathbb{R}_{+}\right), a(t)>0$. If $w(t, s)$ and its partial derivative $\partial w(t, s) / \partial t$ are real-valued nonnegative continuous functions for $t, s \in \mathbb{R}_{+}$with $s \leq t$, then the inequality

$$
u^{p}(t) \leq a(t)+b(t) \int_{0}^{t} w(t, s)\left[f(s) u^{p}(s)+g(s) u^{q}(s)+m(s)\right] \mathrm{d} s, \quad t \in \mathbb{R}_{+},
$$

implies

$$
u(t) \leq a^{1 / p}(t)+\frac{1}{p} a^{1 / p-1}(t) b(t) \int_{0}^{t} \bar{G}(s) \exp \left(\int_{s}^{t} \bar{A}(\tau) d \tau\right) \mathrm{d} s, \quad t \in \mathbb{R}_{+},
$$

where

$$
\begin{aligned}
\bar{A}(t)= & w(t, t) b(t)\left(f(t)+\frac{q}{p} a^{q / p-1}(t) g(t)\right) \\
& +\int_{0}^{t} \frac{\partial w(t, s)}{\partial t} b(s)\left(f(s)+\frac{q}{p} a^{q / p-1}(s) g(s)\right) \mathrm{d} s, \\
\bar{G}(t)= & w(t, t)\left[a(t) f(t)+g(t) a^{q / p}(t)+m(t)\right] \\
& +\int_{0}^{t} \frac{\partial w(t, s)}{\partial t}\left[a(s) f(s)+g(s) a^{q / p}(s)+m(s)\right] \mathrm{d} s .
\end{aligned}
$$

Corollary 2.13. Let $\mathbb{T}=\mathbb{Z}$ and assume that $a(t)>0, u(t), b(t), f(t), g(t)$ and $m(t)$ are nonnegative functions defined for $t \in \mathbb{N}_{0}$. If $w(t, s)$ and $\Delta_{1} w(t, s)$ are real-valued nonnegative functions for $t, s \in$ $\mathbb{N}_{0}$ with $s \leq t$, then the inequality

$$
u^{p}(t) \leq a(t)+b(t) \sum_{s=0}^{t-1} w(t, s)\left[f(s) u^{p}(s)+g(s) u^{q}(s)+m(s)\right], \quad t \in \mathbb{N}_{0},
$$

implies

$$
u(t) \leq a^{1 / p}(t)+\frac{1}{p} a^{1 / p-1}(t) b(t) \sum_{s=0}^{t-1} \tilde{G}(s) \prod_{\tau=s+1}^{t-1}(1+\widetilde{A}(\tau)), \quad t \in \mathbb{N}_{0}
$$


where $\boldsymbol{\Delta}_{1} w(t, s)=w(t+1, s)-w(t, s)$ for $t, s \in \mathbb{N}_{0}$ with $s \leq t$,

$$
\begin{aligned}
\tilde{A}(t)= & w(t+1, t) b(t)\left(f(t)+\frac{q}{p} a^{q / p-1}(t) g(t)\right) \\
& +\sum_{s=0}^{t-1} \Delta_{1} w(t, s) b(s)\left(f(s)+\frac{q}{p} a^{q / p-1}(s) g(s)\right), \\
\tilde{G}(t)= & w(t+1, t)\left[a(t) f(t)+g(t) a^{q / p}(t)+m(t)\right] \\
& +\sum_{s=0}^{t-1} \Delta_{1} w(t, s)\left[a(s) f(s)+g(s) a^{q / p}(s)+m(s)\right] .
\end{aligned}
$$

Corollary 2.14. Suppose that $u(t), a(t)$, and $w(t, s)$ are defined as in Theorem 2.11. If $a(t)$ is nondecreasing for $t \in \mathbb{T}^{\kappa}$, then

$$
u^{p}(t) \leq a(t)+\int_{t_{0}}^{t} w(t, \tau) u^{q}(\tau) \Delta \tau, \quad t \in \mathbb{T}^{\kappa}
$$

implies

$$
u(t) \leq a^{1 / p}(t)\left[1-\frac{1}{q}+\frac{1}{q} e \widetilde{\sim}\left(t, t_{0}\right)\right], \quad t \in \mathbb{T}^{\kappa},
$$

where

$$
\widetilde{\bar{A}}(t)=\frac{q}{p}\left(w(\sigma(t), t) a^{1 / p-1}(t)+\int_{t_{0}}^{t} w^{\Delta}(t, \tau) a^{1 / p-1}(\tau) \Delta \tau\right) .
$$

Proof. Letting $b(t)=1, f(t)=0, g(t)=1$, and $m(t)=0$ in Theorem 2.11, we obtain

$$
\begin{aligned}
A(t) & =\frac{q}{p}\left(w(\sigma(t), t) a^{1 / p-1}(t)+\int_{t_{0}}^{t} w^{\Delta}(t, \tau) a^{1 / p-1}(\tau) \Delta \tau\right):=\tilde{\bar{A}}(t), \\
G(t) & =w(\sigma(t), t) a^{q / p}(t)+\int_{t_{0}}^{t} w^{\Delta}(t, \tau) a^{q / p}(\tau) \Delta \tau \\
& \leq a(t)\left(w(\sigma(t), t) a^{1 / p-1}(t)+\int_{t_{0}}^{t} w^{\Delta}(t, \tau) a^{1 / p-1}(\tau) \Delta \tau\right) \\
& =\frac{p}{q} a(t) \tilde{\bar{A}}(t), \quad t \in \mathbb{T}^{\mathcal{K}},
\end{aligned}
$$


where the inequality holds because $a(t)$ is nondecreasing for $t \in \mathbb{T}^{\kappa}$. Therefore, using Theorem 2.11 and noting (2.46), we easily have

$$
\begin{aligned}
u(t) & \leq a^{1 / p}(t)+\frac{1}{p} a^{1 / p-1}(t) \int_{t_{0}}^{t} e_{A}(t, \sigma(\tau)) G(\tau) \Delta(\tau) \\
& \leq a^{1 / p}(t)+\frac{1}{p} a^{1 / p-1}(t) \int_{t_{0}}^{t} e_{\tilde{\bar{A}}}(t, \sigma(\tau)) \frac{p}{q} a(\tau) \tilde{\bar{A}}(\tau) \Delta \tau \\
& \leq a^{1 / p}(t)+\frac{1}{q} a^{1 / p}(t) \int_{t_{0}}^{t} e_{\tilde{\bar{A}}}(t, \sigma(\tau)) \tilde{\bar{A}}(\tau) \Delta \tau \\
& =a^{1 / p}(t)\left[1+\frac{1}{q}\left(e_{\tilde{\bar{A}}}\left(t, t_{0}\right)-e_{\tilde{\bar{A}}}(t, t)\right)\right] \\
& =a^{1 / p}(t)\left[1-\frac{1}{q}+\frac{1}{q} e_{\tilde{\bar{A}}}\left(t, t_{0}\right)\right], \quad t \in \mathbb{T}^{\kappa} .
\end{aligned}
$$

The proof of Corollary 2.14 is complete.

By Theorem 2.11, we can establish the following more general result.

Theorem 2.15. Assume that $u, a, b, f, g_{i}, m \in C_{\mathrm{rd}}, a(t)>0, u(t), b(t), f(t), g_{i}(t)$, and $m(t)$ are nonnegative, $i=1,2, \ldots, n$, and there exists a series of positive real numbers $q_{1}, q_{2}, \ldots, q_{n}$ such that $p \geq q_{i}>0, i=1,2, \ldots, n$. If $w(t, s)$ is defined as in Lemma 2.2 such that $w(t, s) \geq 0$ and $w^{\Delta}(t, s) \geq 0$ for $t, s \in \mathbb{T}$ with $s \leq t$, then

$$
u^{p}(t) \leq a(t)+b(t) \int_{t_{0}}^{t} w(t, \tau)\left[f(\tau) u^{p}(\tau)+\sum_{i=1}^{n} g_{i}(\tau) u^{q_{i}}(\tau)+m(\tau)\right] \Delta \tau, \quad t \in \mathbb{T}^{\kappa},
$$

implies

$$
u(t) \leq a^{1 / p}(t)+\frac{1}{p} a^{1 / p-1}(t) b(t) \int_{t_{0}}^{t} e_{A^{*}}(t, \sigma(\tau)) G^{*}(\tau) \Delta(\tau), \quad t \in \mathbb{T}^{\mathcal{K}},
$$

where

$$
\begin{aligned}
A^{*}(t)= & w(\sigma(t), t) b(t)\left(f(t)+\sum_{i=1}^{n} \frac{q_{i}}{p} a^{q_{i} / p-1}(t) g_{i}(t)\right) \\
& +\int_{t_{0}}^{t} w^{\Delta}(t, \tau) b(\tau)\left(f(\tau)+\sum_{i=1}^{n} \frac{q_{i}}{p} a^{q_{i} / p-1}(\tau) g_{i}(\tau)\right) \Delta \tau, \\
G^{*}(t)= & w(\sigma(t), t)\left[a(t) f(t)+\sum_{i=1}^{n} g_{i}(t) a^{q_{i} / p}(t)+m(t)\right] \\
& +\int_{t_{0}}^{t} w^{\Delta}(t, \tau)\left[a(\tau) f(\tau)+\sum_{i=1}^{n} g_{i}(\tau) a^{q_{i} / p}(\tau)+m(\tau)\right] \Delta \tau .
\end{aligned}
$$


Theorem 2.16. Let $u, a, r \in C_{\mathrm{rd}}, u(t)$ and $r(t)$ be nonnegative, $a(t)>0$, and $a(t)$ be nondecreasing. Assume that there exists a series of positive real numbers $q_{1}, q_{2}, \ldots, q_{n}$ such that $p \geq q_{i}>0, i=$ $1,2, \ldots, n$. If $S_{i}: \mathbb{T}^{\kappa} \times \mathbb{R}_{+} \rightarrow \mathbb{R}_{+}$is a continuous function such that

$$
0 \leq S_{i}\left(t, x_{i}\right)-S_{i}\left(t, y_{i}\right) \leq H_{i}\left(t, y_{i}\right)\left(x_{i}-y_{i}\right)
$$

for $t \in \mathbb{T}^{\mathcal{K}}$ and $x_{i} \geq y_{i} \geq 0, i=1,2, \ldots, n$, where $H_{i}: \mathbb{T}^{\mathcal{K}} \times \mathbb{R}_{+} \rightarrow \mathbb{R}_{+}$is a nonnegative continuous function, $i=1,2, \ldots, n$, then

$$
u^{p}(t) \leq a(t)+\int_{t_{0}}^{t} r(\tau) u^{p}(\tau) \Delta \tau+\sum_{i=1}^{n} \int_{t_{0}}^{t} S_{i}\left(\tau, u^{q_{i}}(\tau)\right) \Delta \tau, \quad t \in \mathbb{T}^{\kappa}
$$

implies

$$
u(t) \leq R^{1 / p}(t)\left[a^{1 / p}(t)+\frac{1}{p} a^{1 / p-1}(t) L(t) e_{J}\left(t, t_{0}\right)\right], \quad t \in \mathbb{T}^{\kappa},
$$

where

$$
\begin{aligned}
& R(t)=e_{r}\left(t, t_{0}\right), \\
& L(t)=\sum_{i=1}^{n} \int_{t_{0}}^{t} S_{i}\left(\tau, R^{q_{i} / p}(\tau) a^{q_{i} / p}(\tau)\right) \Delta \tau, \\
& J(t)=\sum_{i=1}^{n} \frac{q_{i}}{p} H_{i}\left(t, R^{q_{i} / p}(t) a^{q_{i} / p}(t)\right) R^{q_{i} / p}(t) a^{q_{i} / p-1}(t) .
\end{aligned}
$$

Proof. Let

$$
v(t)=\sum_{i=1}^{n} \int_{t_{0}}^{t} S_{i}\left(\tau, u^{q_{i}}(\tau)\right) \Delta \tau, \quad z(t)=a(t)+v(t), \quad t \in \mathbb{T}^{\kappa} .
$$

Then (2.52) can be restated as

$$
u^{p}(t) \leq z(t)+\int_{t_{0}}^{t} r(\tau) u^{p}(\tau) \Delta \tau, \quad t \in \mathbb{T}^{\kappa}
$$

It is easy to see that $z(t) \in \mathrm{C}_{\mathrm{rd}}, z(t)>0$, and $z(t)$ is nondecreasing. Using Lemma 2.4, from (2.58), we have

$$
u^{p}(t) \leq R(t) z(t), \quad t \in \mathbb{T}^{\kappa}
$$

where $R(t)$ is defined as in (2.54). It follows from (2.57) and (2.59) that

$$
u^{p}(t) \leq R(t)[a(t)+v(t)] .
$$


Using Lemma 2.1 to the above inequality, we obtain

$$
\begin{aligned}
u(t) & \leq R^{1 / p}(t)[a(t)+v(t)]^{1 / p} \\
& \leq R^{1 / p}(t)\left[a^{1 / p}(t)+\frac{1}{p} a^{1 / p-1}(t) v(t)\right], \\
u^{q_{i}}(t) & \leq R^{q_{i} / p}(t)[a(t)+v(t)]^{q_{i} / p} \\
& \leq R^{q_{i} / p}(t)\left[a^{q_{i} / p}(t)+\frac{q_{i}}{p} a^{q_{i} / p-1}(t) v(t)\right], \quad t \in \mathbb{T}^{\kappa} .
\end{aligned}
$$

Noting the hypotheses on $S_{i}$, from (2.62), we get

$$
\begin{aligned}
v(t) \leq \sum_{i=1}^{n} \int_{t_{0}}^{t} S_{i}\left(\tau, R^{q_{i} / p}(\tau)\left[a^{q_{i} / p}(\tau)+\frac{q_{i}}{p} a^{q_{i} / p-1}(\tau) v(\tau)\right]\right) \Delta \tau \\
=\sum_{i=1}^{n} \int_{t_{0}}^{t}\left\{S_{i}\left(\tau, R^{q_{i} / p}(\tau)\left[a^{q_{i} / p}(\tau)+\frac{q_{i}}{p} a^{q_{i} / p-1}(\tau) v(\tau)\right]\right)\right. \\
\left.\quad-S_{i}\left(\tau, R^{q_{i} / p}(\tau) a^{q_{i} / p}(\tau)\right)\right\} \Delta \tau+\sum_{i=1}^{n} \int_{t_{0}}^{t} S_{i}\left(\tau, R^{q_{i} / p}(\tau) a^{q_{i} / p}(\tau)\right) \Delta \tau \\
\leq L(t)+\sum_{i=1}^{n} \int_{t_{0}}^{t} H_{i}\left(\tau, R^{q_{i} / p}(\tau) a^{q_{i} / p}(\tau)\right) R^{q_{i} / p}(\tau) \frac{q_{i}}{p} a^{q_{i} / p-1}(\tau) v(\tau) \Delta \tau, \quad t \in \mathbb{T}^{\mathcal{K}},
\end{aligned}
$$

where $L(t)$ is defined by (2.55). Clearly, $L(t) \geq 0$ and $L(t)$ are nondecreasing. Therefore, for any $\varepsilon>0$, from (2.63), we obtain

$$
\frac{v(t)}{L(t)+\varepsilon} \leq 1+\sum_{i=1}^{n} \int_{t_{0}}^{t} H_{i}\left(\tau, R^{q_{i} / p}(\tau) a^{q_{i} / p}(\tau)\right) R^{q_{i} / p}(\tau) \frac{q_{i}}{p} a^{q_{i} / p-1}(\tau) \frac{v(\tau)}{L(\tau)+\varepsilon} \Delta \tau, \quad t \in \mathbb{T}^{\kappa} .
$$

Let

$$
\psi(t)=\frac{v(t)}{L(t)+\varepsilon}, \quad t \in \mathbb{T}^{\kappa},
$$

and define $k(t)$ by the right hand of (2.64). Then $k(t)>0, k\left(t_{0}\right)=1, \psi(t) \leq k(t)$, and

$$
\begin{aligned}
k^{\Delta}(t) & =\sum_{i=1}^{n} H_{i}\left(t, R^{q_{i} / p}(t) a^{q_{i} / p}(t)\right) R^{q_{i} / p}(t) \frac{q_{i}}{p} a^{q_{i} / p-1}(t) \psi(t) \\
& \leq \sum_{i=1}^{n} H_{i}\left(t, R^{q_{i} / p}(t) a^{q_{i} / p}(t)\right) R^{q_{i} / p}(t) \frac{q_{i}}{p} a^{q_{i} / p-1}(t) k(t) \\
& =k(t) J(t), \quad t \in \mathbb{T}^{\kappa},
\end{aligned}
$$


where $J(t)$ is defined by (2.56). Using Lemma 2.3 and noting $k\left(t_{0}\right)=1$, from (2.66), we have

$$
k(t) \leq e_{J}\left(t, t_{0}\right), \quad t \in \mathbb{T}^{\kappa} .
$$

Therefore,

$$
v(t) \leq(L(t)+\varepsilon) e_{J}\left(t, t_{0}\right), \quad t \in \mathbb{T}^{\kappa}
$$

It follows from (2.61) and (2.68) that

$$
u(t) \leq R^{1 / p}(t)\left[a^{1 / p}(t)+\frac{1}{p} a^{1 / p-1}(t)(L(t)+\varepsilon) e_{J}\left(t, t_{0}\right)\right], \quad t \in \mathbb{T}^{\kappa} .
$$

Letting $\varepsilon \rightarrow 0$ in (2.69), we immediately obtain the desired inequality (2.53). This completes the proof of Theorem 2.16.

Corollary 2.17. Let $\mathbb{T}=\mathbb{R}, u, a, r \in C\left(\mathbb{R}_{+}, \mathbb{R}_{+}\right), a(t)>0$, and $a(t)$ be nondecreasing. Assume that there exists a series of positive real numbers $q_{1}, q_{2}, \ldots, q_{n}$ such that $p \geq q_{i}>0, i=1,2, \ldots, n$. If $S_{i}: \mathbb{R}_{+} \times \mathbb{R}_{+} \rightarrow \mathbb{R}_{+}$is a continuous function such that

$$
0 \leq S_{i}\left(t, x_{i}\right)-S_{i}\left(t, y_{i}\right) \leq H_{i}\left(t, y_{i}\right)\left(x_{i}-y_{i}\right)
$$

for $t \in \mathbb{R}_{+}$and $x_{i} \geq y_{i} \geq 0, i=1,2, \ldots, n$, where $H_{i}: \mathbb{R}_{+} \times \mathbb{R}_{+} \rightarrow \mathbb{R}_{+}$is a continuous function, $i=1,2, \ldots, n$, then

$$
u^{p}(t) \leq a(t)+\int_{0}^{t} r(\tau) u^{p}(\tau) \mathrm{d} \tau+\sum_{i=1}^{n} \int_{0}^{t} S_{i}\left(\tau, u^{q_{i}}(\tau)\right) \mathrm{d} \tau, \quad t \in \mathbb{R}_{+}
$$

implies

$$
u(t) \leq \bar{R}^{1 / p}(t)\left[a^{1 / p}(t)+\frac{1}{p} a^{1 / p-1}(t) \bar{L}(t) \exp \left(\int_{0}^{t} \bar{J}(s) \mathrm{d} s\right)\right], \quad t \in \mathbb{R}_{+}
$$

where $J(t)$ is defined as in (2.56),

$$
\begin{aligned}
& \bar{R}(t)=\exp \left(\int_{0}^{t} r(s) \mathrm{d} s\right) \\
& \bar{L}(t)=\sum_{i=1}^{n} \int_{0}^{t} S_{i}\left(\tau, \bar{R}^{q_{i} / p}(\tau) a^{q_{i} / p}(\tau)\right) \mathrm{d} \tau, \\
& \bar{J}(t)=\sum_{i=1}^{n} \frac{q_{i}}{p} H_{i}\left(t, \bar{R}^{q_{i} / p}(t) a^{q_{i} / p}(t)\right) \bar{R}^{q_{i} / p}(t) a^{q_{i} / p-1}(t) .
\end{aligned}
$$


Corollary 2.18. Let $\mathbb{T}=\mathbb{Z}, a(t)>0, a(t)$ be nondecreasing, $u(t)$ and $r(t)$ be nonnegative functions defined for $t \in \mathbb{N}_{0}$. Assume that there exists a series of positive real numbers $q_{1}, q_{2}, \ldots, q_{n}$ such that $p \geq q_{i}>0, i=1,2, \ldots, n$. If $S_{i}: \mathbb{N}_{0} \times \mathbb{R}_{+} \rightarrow \mathbb{R}_{+}$such that

$$
0 \leq S_{i}\left(t, x_{i}\right)-S_{i}\left(t, y_{i}\right) \leq H_{i}\left(t, y_{i}\right)\left(x_{i}-y_{i}\right)
$$

for $t \in \mathbb{N}_{0}$ and $x_{i} \geq y_{i} \geq 0, i=1,2, \ldots, n$, where $H_{i}: \mathbb{N}_{0} \times \mathbb{R}_{+} \rightarrow \mathbb{R}_{+}, i=1,2, \ldots, n$, then

$$
u^{p}(t) \leq a(t)+\sum_{\tau=0}^{t-1} r(\tau) u^{p}(\tau)+\sum_{i=1}^{n} \sum_{\tau=0}^{t-1} S_{i}\left(\tau, u^{q_{i}}(\tau)\right), \quad t \in \mathbb{N}_{0}
$$

implies

$$
u(t) \leq \tilde{R}^{1 / p}(t)\left[a^{1 / p}(t)+\frac{1}{p} a^{1 / p-1}(t) \widetilde{L}(t) \prod_{s=0}^{t-1}(1+\widetilde{J}(s))\right], \quad t \in \mathbb{N}_{0}
$$

where $J(t)$ is defined as in (2.56),

$$
\begin{aligned}
& \tilde{R}(t)=\prod_{s=0}^{t-1}(1+r(s)) \\
& \widetilde{L}(t)=\sum_{i=1}^{n} \sum_{\tau=0}^{t-1} S_{i}\left(\tau, \widetilde{R}^{q_{i} / p}(\tau) a^{q_{i} / p}(\tau)\right) \\
& \tilde{J}(t)=\sum_{i=1}^{n} \frac{q_{i}}{p} H_{i}\left(t, \widetilde{R}^{q_{i} / p}(t) a^{q_{i} / p}(t)\right) \tilde{R}^{q_{i} / p}(t) a^{q_{i} / p-1}(t) .
\end{aligned}
$$

Remark 2.19. Using our main results, we can obtain many dynamic inequalities for some peculiar time scales. Due to limited space, their statements are omitted here.

\section{Some Applications}

In this section, we present two applications of our main results.

Example 3.1. Consider the inequality as in (2.25) with $a(t)=\alpha(t+1), b(t)=\alpha\left(t^{2}+1\right), g(t)=$ $t, h(t)=0, p=2, q=1, \alpha=10^{-6}$, and we compute the values of $u(t)$ from (2.25) and also we find the values of $u(t)$ by using the result (2.26). In our computations we use (2.25) and (2.26) as equations and as we see in Table 1 the computation values as in (2.25) are less than the values of the result (2.26).

From Table 1, we easily find that the numerical solution agrees with the analytical solution for some discrete inequalities. The program is written in the programming Matlab 7.0. 
Table 1

\begin{tabular}{lcc}
\hline$t$ & $(2.25)$ & $(2.26)$ \\
\hline 1 & $1.414213562373095 \mathrm{e}-003$ & $1.414213562373095 \mathrm{e}-003$ \\
2 & $2.661293464584210 \mathrm{e}-003$ & $2.910562109546456 \mathrm{e}-003$ \\
5 & $5.486250637546570 \mathrm{e}-002$ & $1.103460932829943 \mathrm{e}-001$ \\
7 & $2.670738191264154 \mathrm{e}-001$ & $5.410171853718061 \mathrm{e}-001$ \\
10 & $1.527219045903506 \mathrm{e}+000$ & $3.137697944498020 \mathrm{e}+000$ \\
12 & $3.720520602864323 \mathrm{e}+000$ & $8.436559692675310 \mathrm{e}+000$ \\
14 & $7.856747926470754 \mathrm{e}+000$ & $2.187361362745254 \mathrm{e}+001$ \\
17 & $1.997586843703775 \mathrm{e}+001$ & $9.900992670086097 \mathrm{e}+001$ \\
20 & $4.331228422296512 \mathrm{e}+001$ & $5.854191578762491 \mathrm{e}+002$ \\
25 & $1.241251179017371 \mathrm{e}+002$ & $2.937887676184530 \mathrm{e}+004$ \\
\hline
\end{tabular}

Example 3.2. Consider the following initial value problem on time scales:

$$
\left(u^{p}(t)\right)^{\Delta}=M(t, u(t)), \quad u\left(t_{0}\right)=\beta, \quad t \in \mathbb{T}^{\mathcal{K}},
$$

where $p \geq 1$ and $\beta \neq 0$ are constants, and $M: \mathbb{T}^{\kappa} \times \mathbb{R} \rightarrow \mathbb{R}$ is a continuous function.

Assume that

$$
|M(t, u(t))| \leq g(t)\left|u^{q}(t)\right|,
$$

where $g(t)$ is defined as in Corollary 2.6, $0<q \leq p$ is a constant. If $u(t)$ is a solution of IVP (3.1), then

$$
|u(t)| \leq|\beta|\left(1-\frac{1}{q}+\frac{1}{q} e_{V}\left(t, t_{0}\right)\right), \quad t \in \mathbb{T}^{\kappa},
$$

where

$$
V(t)=\frac{q}{p}|\beta|^{q-p} g(t), \quad t \in \mathbb{T}^{\kappa}
$$

In fact, the solution $u(t)$ of IVP (3.1) satisfies the following equation:

$$
u^{p}(t)=\beta^{p}+\int_{t_{0}}^{t} M(\tau, u(\tau)) \Delta \tau, \quad t \in \mathbb{T}^{\kappa} .
$$

Using the assumption (3.2), from (3.5), we have

$$
|u(t)|^{p} \leq|\beta|^{p}+\int_{t_{0}}^{t} g(\tau)|u(\tau)|^{q} \Delta \tau, \quad t \in \mathbb{T}^{\kappa} .
$$

Now a suitable application of Corollary 2.6 to (3.6) yields (3.2). 


\section{Acknowledgments}

This work is supported by the Natural Science Foundation of Shandong Province (Y2007A08), the National Natural Science Foundation of China $(60674026,10671127)$, China Postdoctoral Science Foundation Funded Project (20080440633), Shanghai Postdoctoral Scientific Program (09R21415200), the Project of Science and Technology of the Education Department of Shandong Province (J08LI52), and the Doctoral Foundation of Binzhou University (2006Y01).

\section{References}

[1] S. Hilger, "Analysis on measure chains-a unified approach to continuous and discrete calculus," Results in Mathematics, vol. 18, no. 1-2, pp. 18-56, 1990.

[2] M. Bohner and A. Peterson, Dynamic Equations on Time Scales: An Introduction with Application, Birkhäuser, Boston, Mass, USA, 2001.

[3] M. Bohner and A. Peterson, Advances in Dynamic Equations on Time Scales, Birkhäuser, Boston, Mass, USA, 2003.

[4] M. Bohner, L. Erbe, and A. Peterson, "Oscillation for nonlinear second order dynamic equations on a time scale," Journal of Mathematical Analysis and Applications, vol. 301, no. 2, pp. 491-507, 2005.

[5] L. Erbe, A. Peterson, and C. Tisdell, "Existence of solutions to second-order BVPs on time scales," Applicable Analysis, vol. 84, no. 10, pp. 1069-1078, 2005.

[6] H.-R. Sun and W.-T. Li, "Positive solutions of second-order half-linear dynamic equations on time scales," Applied Mathematics and Computation, vol. 158, no. 2, pp. 331-344, 2004.

[7] Y. Xing, M. Han, and G. Zheng, "Initial value problem for first-order integro-differential equation of Volterra type on time scales," Nonlinear Analysis: Theory, Methods $\mathcal{E}$ Applications, vol. 60, no. 3, pp. 429-442, 2005.

[8] S. H. Saker, R. P. Agarwal, and D. O’Regan, “Oscillation results for second-order nonlinear neutral delay dynamic equations on time scales," Applicable Analysis, vol. 86, no. 1, pp. 1-17, 2007.

[9] R. Agarwal, M. Bohner, and A. Peterson, "Inequalities on time scales: a survey," Mathematical Inequalities $\mathcal{E}$ Applications, vol. 4, no. 4, pp. 535-557, 2001.

[10] E. Akin-Bohner, M. Bohner, and F. Akin, "Pachpatte inequalities on time scales," Journal of Inequalities in Pure and Applied Mathematics, vol. 6, article 6, 2005.

[11] W. N. Li, "Some new dynamic inequalities on time scales," Journal of Mathematical Analysis and Applications, vol. 319, no. 2, pp. 802-814, 2006.

[12] F.-H. Wong, C.-C. Yeh, and C.-H. Hong, "Gronwall inequalities on time scales," Mathematical Inequalities \& Applications, vol. 9, no. 1, pp. 75-86, 2006.

[13] D. B. Pachpatte, "Explicit estimates on integral inequalities with time scale," Journal of Inequalities in Pure and Applied Mathematics, vol. 7, no. 4, article 143, 2006.

[14] W. N. Li and W. Sheng, "Some nonlinear dynamic inequalities on time scales," Proceedings of the Indian Academy of Sciences. Mathematical Sciences, vol. 117, no. 4, pp. 545-554, 2007.

[15] D. S. Mitrinović, Analytic Inequalities, Springer, New York, NY, USA, 1970. 